1 Instituto Federal de Educação, Ciência e Tecnologia do Ceará (IFCE) - Tianguá (CE), Brasil. Universidade Federal do Ceará (UFC) - Sobral (CE), Brasil.

emanoelavelar@gmail.com

2 Universidade Federal do Ceará (UFC), Mestrado

Acadêmico em Saúde da Família (Masf) - Sobral

(CE), Brasil. Universidade Estadual Vale do Acaraú (UVA) - Sobral (CE), Brasil. cibellyaliny@gmail.com

3 Universidade Federal do Ceará (UFC), Mestrado Acadêmico em Saúde da Família (Masf) - Sobral (CE), Brasil. Universidade Estadual Vale do Acaraú (UVA) - Sobral (CE), Brasil. elianyy@hotmail.com

4 Universidade Federal do Paraná (UFPR), Programa de Pós-graduação em Enfermagem - Curitiba (PR), Brasil.

mrlacerda55@gmail.com

\section{Grau de sobrecarga dos cuidadores de idosos atendidos em domicílio pela Estratégia Saúde da Família}

\author{
Overload degree of caregivers of elderly assisted at home by the \\ Family Health Strategy \\ Emanoel Avelar Muniz', Cibelly Aliny Siqueira Lima Freitas², Eliany Nazaré Oliveira³, Maria \\ Ribeiro Lacerda 4
}

RESUMO Objetivou-se conhecer as características sociodemográficas, de saúde e o grau de sobrecarga dos cuidadores de idosos atendidos em domicílio pela Estratégia Saúde da Família de Sobral (CE). Pesquisa de abordagem quantitativa do tipo descritiva, foram entrevistados 60 cuidadores de idosos e utilizou-se a escala de Zarit. Os cuidadores são de todas as idades, inclusive tendo mais de 60 anos (26,7\%); do sexo feminino (90\%); portadores de hipertensão $(33,3 \%)$ e diabetes (16,7\%); e apresentam sobrecarga leve (81,7\%). Identificou-se a existência de idosos cuidando de idosos e fragilidade nas ações de apoio às famílias, necessitando de novas estratégias de cuidado e do direcionamento de políticas.

PALAVRAS-CHAVE Cuidadores. Saúde do idoso. Assistência domiciliar. Estratégia Saúde da Família.

\begin{abstract}
This study aimed to know the sociodemographic characteristics, health and the degree of overload on caregivers of patients attended at home by the Family Health Strategy of Sobral (CE). In this quantitative approach research of descriptive type, 60 caregivers of the elderly were interviewed an it was used Zarit scale. Caregivers are of all ages, including those who have more than 60 years (26.7\%), female (90\%), hypertensive (33.3\%), and diabetes (16.7\%); and present light overload (81.7\%). It was identified the existence of older people caring for the elder$l y$ and fragility in supporting actions to families, requiring new care strategies and direction of policies.
\end{abstract}

KEYWORDS Caregivers. Health of the elderly. Home nursing. Family Health Strategy. 


\section{Introdução}

O crescimento da população idosa é um fenômeno mundial e, no Brasil, sofre modificações radicais e bastante aceleradas. A cada ano, 650 mil novos idosos são incorporados à população brasileira, a maior parte com doenças crônicas e alguns com limitações funcionais. Um dos resultados dessa dinâmica é a maior procura dos idosos por serviços de saúde (VERAS, 2009).

Nesse contexto, a Estratégia Saúde da Família (ESF) foi planejada para reorientar a atenção à saúde da população, fomentando a qualidade de vida, por exemplo, mediante a promoção do envelhecimento saudável. Como o envelhecimento não é um processo homogêneo, as necessidades e demandas dos idosos variam, sendo preciso fortalecer o trabalho em rede para contemplar a atenção aos idosos saudáveis e atender àqueles com diferentes graus de incapacidade ou enfermidade, inclusive nos domicílios (MOTTA; AGUIAR; CALDAS, 2011).

O Ministério da Saúde (BRASIL, 2006B) define a Atenção Domiciliar (AD) às pessoas idosas como um conjunto de ações realizadas por uma equipe interdisciplinar no domicílio do usuário/família, a partir do diagnóstico da realidade em que estão inseridos, de seus potenciais e limitações. Articulam promoção, prevenção, diagnóstico, tratamento e reabilitação, favorecendo assim, o desenvolvimento e adaptação de suas funções de maneira a restabelecer sua independência e a preservação de sua autonomia.

A primeira condição para que ocorra a assistência domiciliar é o consentimento da família em relação à presença do cuidador, que é a pessoa que presta diretamente os cuidados ao idoso, de maneira contínua e/ou regular, podendo, ou não, ser alguém da família. Propõe-se que o cuidador seja orientado pela equipe de saúde nos cuidados a serem realizados diariamente no próprio domicílio. As atribuições devem ser pactuadas entre equipe, família e cuidador, democratizando saberes, poderes e responsabilidades. (BRASIL, 2012).

Nesse sentido Gratão et al. (2013) destacam que o suporte aos cuidadores representa novo desafio para o sistema de saúde brasileiro, justificando a necessidade de estudos sobre essa temática principalmente no que se refere ao conhecimento das causas que levam o cuidador a adoecer e, consequentemente, das necessidades de saúde dessa população.

Assim, por conta da potencialidade da AD para ampliar a integralidade na assistência à saúde e, do grande número de idosos e familiares/cuidadores que não se sentem amparados pelo sistema/equipe de saúde objetiva-se conhecer as características sociodemográficas e de saúde, bem como o grau de sobrecarga dos cuidadores de idosos em AD na ESF de Sobral (CE). Esta pesquisa mostra-se relevante haja vista a possibilidade de traçar um perfil dos cuidadores de idosos, identificar necessidades e possibilidades para a construção de uma política de saúde que contemple essa população.

\section{Métodos}

Esta pesquisa é de abordagem quantitativa do tipo descritiva. Foi eleito como campo de pesquisa o município de Sobral, principal cidade do noroeste do Ceará, localizada a $238 \mathrm{~km}$ de Fortaleza (CE), contando com uma área de aproximadamente $2.123 \mathrm{~km}^{2} \mathrm{e}$ uma população de 212.718 habitantes (SOBRAL, 2012).

A ESF de Sobral (CE) possui 34 Centros de Saúde da Família (CSF) para todo o município, sendo 20 situados na zona urbana e 14 na zona rural. As equipes da ESF totalizam 57 , sendo que 41 atuam na sede e 16 na zona rural. Atualmente, a ESF do município está dividida em quatro macro áreas de saúde, de acordo com as características de cada território.

Reconhecendo a grande quantidade e 
diversidade de territórios da ESF de Sobral (CE), foi utilizada a amostragem intencional, elegendo como critério de escolha a macro área que possuía o maior número de idosos de acordo com o Sistema de Informação da Atenção Básica (Siab) de 2013. Sendo assim, esta pesquisa foi realizada nos territórios da macro área II, incluindo $6 \mathrm{CSF}$ da sede do município que possuía uma população de idosos de aproximadamente 7 mil pessoas. A coleta de informações foi desenvolvida no período de setembro de 2014 a março de 2015.

Partindo da informação obtida junto às gerentes dos CSF, de que os idosos domiciliados e/ou acamados representavam cerca de $10 \%$ da população de idosos atendidos pela ESF, a amostra de sujeitos do estudo foi de 60 familiares/cuidadores principais, responsáveis pelo cuidado em domicílio de idosos em $\mathrm{AD}$ na ESF identificados pelas respectivas equipes representando aproximadamente $9 \%$ do total de cuidadores da macro área II. Os sujeitos aceitaram participar da pesquisa após conhecerem e assinarem o Termo de Consentimento Livre e Esclarecido (TCLE). Os instrumentos de coleta de dados utilizados foram a entrevista semiestruturada e a escala de sobrecarga do cuidador, de Zarit.

A entrevista semiestruturada continha questões sobre a situação sociodemográfica dos cuidadores; problemas de saúde, uso de álcool, fumo e/ou drogas ilícitas; consumo de medicações; prática de atividades físicas; grau de parentesco com o idoso; tempo de exercício da atividade de cuidador; compartilhamento das tarefas de cuidado; recebimento de auxílio financeiro para exercer a atividade; e realização de treinamento ou capacitação para o cuidado.

A avaliação da sobrecarga dos cuidadores de Zarit serviu para avaliar se os cuidadores de idosos estão sobrecarregados. Foi aplicada para o cuidador principal - pessoa que mais ajuda, e realizada sem a presença do idoso.

Para a organização e a análise dos dados, utilizou-se a estatística descritiva, distribuindo em porcentagens as principais características sociodemográficas e de saúde dos cuidadores além do resultado da escala de Zarit.

Adotaram-se as recomendações éticas para pesquisa envolvendo seres humanos contidas na Resolução 466/2012 do Conselho Nacional de Saúde (CNS), obtendo-se a aprovação no Comitê de Ética em Pesquisa da Universidade Estadual Vale do Acaraú (UVA), sob o $\mathrm{n}^{\mathrm{o}} 31215114.9 .0000 .5053$ do Certificado de Apresentação para Apreciação Ética (CAAE).

Este artigo é um recorte da dissertação intitulada 'Atenção domiciliar na Estratégia Saúde da Família: análise das perspectivas de idosos, cuidadores e profissionais', apresentada ao Mestrado Acadêmico em Saúde da Família (Masf), da Universidade Federal do Ceará (UFC) - Campus Sobral.

\section{Resultados e discussão}

Considerando a importância de conhecer os sujeitos envolvidos na $\mathrm{AD}$, no âmbito da ESF, será apresentada uma descrição das principais características sociodemográficas e de saúde dos familiares/cuidadores principais dos idosos, além de fatores inerentes ao cuidado oferecido, e avaliação do grau de sobrecarga do cuidador, conforme a escala de Zarit. 
Tabela 1. Características sociodemográficas dos cuidadores de idosos em Atenção Domiciliar, na macro área II, da Estratégia Saúde da Família de Sobral, Ceará, 2015

\begin{tabular}{|c|c|c|}
\hline Características & Quantidade & Porcentagem \\
\hline Idade & $\mathbf{N}$ & $\%$ \\
\hline $20-29$ & 6 & 10 \\
\hline $30-39$ & 12 & 20 \\
\hline $40-49$ & 13 & 21,7 \\
\hline $50-59$ & 13 & 21,7 \\
\hline$>60$ & 16 & 26,7 \\
\hline Sexo & $\mathbf{N}$ & $\%$ \\
\hline Feminino & 54 & 90 \\
\hline Masculino & 6 & 10 \\
\hline Escolaridade (anos) & $\mathbf{N}$ & $\%$ \\
\hline Nenhum & 14 & 23,3 \\
\hline 1 a 4 & 21 & 35 \\
\hline 5 a 8 & 11 & 18,3 \\
\hline 9 a 11 & 6 & 10 \\
\hline$>11$ & 8 & 13,3 \\
\hline Raça & $\mathbf{N}$ & $\%$ \\
\hline Parda & 47 & 78,3 \\
\hline Branca & 6 & 10 \\
\hline Preta & 7 & 11,7 \\
\hline Religião & $\mathbf{N}$ & $\%$ \\
\hline Católica & 46 & 76,7 \\
\hline Evangélica & 8 & 13,3 \\
\hline Testemunha de Jeová & 2 & 3,3 \\
\hline Não tem & 4 & 6,6 \\
\hline Situação conjugal & $\mathbf{N}$ & $\%$ \\
\hline Solteiro & 28 & 46,7 \\
\hline Casado & 16 & 26,7 \\
\hline União estável & 10 & 16,7 \\
\hline Divorciado/Separado & 5 & 8,3 \\
\hline Viúvo & 1 & 1,7 \\
\hline Total & 60 & 100 \\
\hline
\end{tabular}

Fonte: Elaboração própria. 
Entre as principais características sociodemográficas identificadas nos cuidadores de idosos que recebem $\mathrm{AD}$ pela $\mathrm{ESF}$ de Sobral (CE), destacam-se pessoas de todas as idades, especialmente com mais de 60 anos
(26,7\%), ou seja, idosos cuidando de outros idosos; sexo feminino (90\%); baixa escolaridade, um a quatro anos de estudo (35\%), cor parda (78,3\%); religião católica $(76,7 \%)$; e solteiros $(46,7 \%)$.

Tabela 2. Características da situação de saúde dos cuidadores de idosos em Atenção Domiciliar, na macro área II, da Estratégia Saúde da Família de Sobral, Ceará, 2015

\begin{tabular}{|c|c|c|}
\hline Características & Quantidade & Porcentagem \\
\hline Problemas de saúde & $\mathbf{N}$ & $\%$ \\
\hline HAS & 20 & 33,3 \\
\hline DM & 10 & 16,7 \\
\hline Osteoarticulares & 11 & 18,3 \\
\hline Dislipidemias & 9 & 15 \\
\hline Psiquiátricos & 7 & 11,7 \\
\hline Neurológicos & 5 & 8,3 \\
\hline Outros & 14 & 23,3 \\
\hline Nenhum & 21 & 35 \\
\hline Medicações utilizadas & $\mathbf{N}$ & $\%$ \\
\hline Anticonvulsivantes & 4 & 6,7 \\
\hline Antidepressivos & 6 & 10 \\
\hline Anti-hipertensivos & 20 & 33,3 \\
\hline Antilipemiantes & 9 & 15 \\
\hline Antiplaquetários & 3 & 5 \\
\hline Antipsicóticos & 3 & 5 \\
\hline Diuréticos & 5 & 8,3 \\
\hline Hipoglicemiantes & 10 & 16,7 \\
\hline Hormônios & 3 & 5 \\
\hline Vitaminas & 4 & 6,7 \\
\hline Outros & 14 & 23,3 \\
\hline Nenhuma & 25 & 41,2 \\
\hline Uso de álcool/fumo & $\mathbf{N}$ & $\%$ \\
\hline Álcool & 6 & 10 \\
\hline Fumo & 10 & 16,7 \\
\hline Nenhum & 47 & 78,3 \\
\hline
\end{tabular}


Tabela 2. (cont.)

\begin{tabular}{lcr}
\hline Realiza atividade física & N & \% \\
\hline Não & 53 & 88,3 \\
Sim & 7 & 11,7 \\
\hline Total & 60 & $\mathbf{1 0 0}$ \\
\hline
\end{tabular}

Fonte: Elaboração própria.

Com relação às características da situação de saúde destacaram-se os consumos de fumo (16,7\%) e álcool (10\%), e, a não realização de atividades físicas regulares $(88,3 \%)$. Entre os problemas de saúde referidos, os mais incidentes foram a Hipertensão Arterial Sistêmica (HAS), em 33,3\% dos cuidadores; o Diabetes Mellitus (DM), em 16,7\%; e os osteoarticulares, em $18,3 \%$. Com relação ao consumo de medicamentos, predominaram os anti-hipertensivos (33,3\%), os hipoglicemiantes (16,7\%) e os antilipemiantes (15\%).

Em pesquisa realizada por Von Kanel et al. (2011) foi identificado risco cardiometabólico particularmente elevado em cuidadores de pacientes com demência que relataram níveis reduzidos de atividade física regular. $\mathrm{O}$ exercício físico reduz o risco de doenças cardiovasculares, mas cuidadores podem ter menos oportunidades de praticar tal atividade.

Tabela 3. Características do trabalho dos cuidadores de idosos em Atenção Domiciliar, na macro área II, da Estratégia Saúde da Família Sobral, Ceará, 2015

\begin{tabular}{lrr}
\hline Características & Quantidade & Porcentagem \\
\hline Cuidador principal & $\mathbf{N}$ & $\%$ \\
\hline Filho & 40 & 66,7 \\
Companheiro & 9 & 15 \\
Neto & 7 & 11,7 \\
Outros & 4 & 6,7 \\
\hline Tempo de cuidador & $\mathbf{N}$ & $\%$ \\
\hline$<1$ ano & 6 & 10 \\
1 ano & 8 & 13,3 \\
2 anos & 8 & 13,3 \\
3 anos & 4 & 6,7 \\
4 anos & 3 & 5 \\
5 anos & 4 & 6,7 \\
$>10$ anos & 12 & 20 \\
\hline
\end{tabular}




\begin{tabular}{|c|c|c|}
\hline Cuidadores secundários & $\mathbf{N}$ & $\%$ \\
\hline Filho & 18 & 30 \\
\hline Neto & 9 & 15 \\
\hline Nora/genro & 7 & 11,7 \\
\hline Irmão & 5 & 8,3 \\
\hline Companheiro & 4 & 6,7 \\
\hline Outros & 10 & 16,7 \\
\hline Nenhum & 22 & 36,7 \\
\hline Auxílio financeiro & $\mathbf{N}$ & $\%$ \\
\hline Não & 44 & 73,3 \\
\hline Sim & 16 & 26,7 \\
\hline Treinamento & $\mathbf{N}$ & $\%$ \\
\hline Não & 50 & 83,3 \\
\hline $\operatorname{sim}$ & 10 & 16,7 \\
\hline Escala de sobrecarga & $\mathbf{N}$ & $\%$ \\
\hline Leve (até 44 pontos) & 49 & 81,7 \\
\hline Moderada ( $>44$ pontos) & 11 & 18,3 \\
\hline Total & 60 & 100 \\
\hline
\end{tabular}

Fonte: Elaboração própria.

No que se refere à execução do cuidado domiciliar, os filhos se destacaram como cuidadores principais $(66,7 \%)$ e secundários (30\%), exercendo essa atividade, na maioria dos casos (55\%), há menos de cinco anos, evidenciando a crescente demanda por novos cuidadores; sem recebimento de auxílio financeiro $(73,3 \%)$, ou, treinamento (83,3\%); e desempenhando esse papel de forma solitária (36,7\%). Quanto ao resultado da escala de sobrecarga de Zarit, apresentaram sobrecarga leve (81,7\%).

Ao comparar os achados da pesquisa com as características sociodemográficas e clínicas dos cuidadores familiares de idosos residentes em Curitiba (PR), verificaram-se as seguintes características: idades entre 50 e 60 anos; sexo feminino; mais de 8 anos de escolaridade; situação conjugal de união estável; crença religiosa; filiação como grau de parentesco; ausência de ocupação profissional; renda familiar de dois a três salários-mínimos; coabitam com o idoso; partilham do cuidado com outros familiares; cuidam do idoso há mais de três anos; realizam acompanhamento médico; e possuem alguma doença, com prevalência de HAS, de depressão e das inflamatórias. Tudo isto, além de apresentarem grau de sobrecarga moderado (SEIMA; LENARDT; CALDAS, 2014).

Assim, notam-se muitas semelhanças entre o grupo de cuidadores de idosos de 
Sobral (CE) e de Curitiba (PR), como também algumas especificidades, provavelmente devidas às melhores condições socioeconômicas e de saúde da população da região Sul do Brasil. Já no estudo realizado por Del Duca, Thumé e Halal (2011), as esposas representaram 39,5\% dos cuidadores de idosos; e os filhos, $23,7 \%$, apontando divergências entre os resultados das pesquisas.

É sabido que a sobrecarga de trabalho está diretamente vinculada ao tipo de doença/ agravo com a qual o cuidador deve lidar. Assim, o fato de ter-se constatado sobrecarga leve neste estudo pode estar relacionado ao perfil do idoso cuidado em casa pela ESF de Sobral (CE). De acordo com Muniz (2015) eles são maioritariamente de idades avançadas e apresentam, predominância de doenças crônicas como HAS, DM e as osteoarticulares, incluindo comorbidades e, grande consumo de medicações. Em relação às Atividades da Vida Diária (AVD) foram considerados dependentes parciais ou independentes.

Em estudo realizado com idosos residentes em uma comunidade rural do Vietnã, verificou-se que: a maioria deles não necessita de apoio nas AVD; idosos que precisam de ajuda total foram encontrados em menor número do que aqueles que precisam de alguma ajuda nas AVD; os filhos e netos foram elencados como principais cuidadores (HOI; THANG; LINDHOLM, 2011). Mendoza-Suárez (2014), em estudo realizado com familiares encarregados do cuidado de pacientes com enfermidades neurológicas, observou que o maior nível de sobrecarga nos cuidadores foi proporcional a maior incapacidade do paciente.

A imensa maioria de cuidadores do sexo feminino vista em Sobral (CE) e em outros estudos destaca a centralidade da mulher enquanto representante dos cuidados na família, e suas consequências paradoxais. Desta forma, Gutierrez e Minayo (2010) lembram que a centralidade conferida à mulher tem importantes implicações políticas, pois traz consigo: (a) a justificação social da ausência e da desresponsabilização do homem na esfera dos cuidados com a saúde; e (b) o aprisionamento da mulher em suas atividades tradicionais, o que é uma forma de permanência da opressão feminina e seu enclausuramento na cena doméstica.

As demandas de trabalho dos cuidadores familiares ocasionam tensão e sintomas depressivos. Os cuidadores que trabalham em tempo integral possuem mais dificuldade para conciliar trabalho e prestação de cuidados. Assim, é necessário identificar grupos de alto risco para a tensão e depressão, devido ao papel de cuidador; além de avaliar cuidadores familiares sobre a flexibilidade do trabalho e incentivá-los a conciliar o trabalho com as responsabilidades de cuidados da família a fim de reduzir a tensão do papel de cuidador (WANG ET AL., 2011).

Com relação ao não recebimento de auxílio financeiro pela maioria dos cuidadores do estudo, Resende e Dias (2008) informam que o conceito de trabalhador presente na Política Nacional de Saúde do Trabalhador (BRASIL, 2004), também considera como trabalhadores - além dos indivíduos remunerados, inseridos no setor formal ou informal de trabalho - aqueles indivíduos que, como os cuidadores familiares de idosos, realizam funções não remuneradas e que fazem parte das atividades econômicas do lar. Assim, é necessário refletir sobre as condições trabalhistas e previdenciárias desses indivíduos, em curto e em longo prazo.

Nesse sentido Simão e Mioto (2016) questionam a viabilidade histórica da AD sem prejuízo do bem-estar da pessoa do cuidado e de sua família. Os autores demonstram a intenção de países latinos de diminuir gastos com o cuidado a pessoas com doenças crônicas ou demandantes de cuidados paliativos e a exigência de um cuidador cotidiano de responsabilidade da família. Isso significa tanto a transferência dos custos do cuidado para as famílias como a produção de significativas alterações na organização e nos modos de vida das famílias, o que implica em abrir mão do trabalho remunerado. 
Além disso, a maioria dos cuidadores no estudo apresentou grau de sobrecarga leve, não foi capacitada para exercer as atividades de cuidado e referiu algum problema de saúde. Stackfleth et al. (2012) afirmam que as atividades de cuidado podem sobrecarregar o cuidador pelo despreparo em relação ao papel que desempenha, e isso poderá causar problemas para sua saúde. Além disso, o excesso de sobrecarga pode comprometer a qualidade do cuidado e interferir nas relações familiares.

Pimenta et al. (2009) destacam que as diferentes dinâmicas do cuidar de um idoso fragilizado poderão influir positivamente ou não no bem-estar de ambos: a pessoa cuidada e a pessoa do cuidador. Por isso, merecem atenção sua vida e saúde de modo particular, por parte dos serviços sociais e de saúde. De Valle-Alonso et al. (2015) propõem a criação de programas sociais preventivos, destinados a desenvolver áreas de lazer para o cuidado de idosos e a apoiar os cuidadores familiares, beneficiando, assim, a qualidade de vida dos idosos.

De acordo com a Política Nacional de Saúde da Pessoa Idosa (BRASIL, 2006A) a família, via de regra, é a executora do cuidado ao idoso, e é necessário estabelecer um suporte qualificado e constante aos responsáveis por esses cuidados, tendo a Atenção Básica, por meio da ESF, um papel fundamental, considerando os cuidadores de idosos parceiros das equipes de saúde na assistência aos idosos dependentes.

Nesse sentido, é importante reforçar o papel das equipes da ESF no treinamento e na supervisão dos cuidadores, visto que o profissional somente vai conquistar a autonomia do cuidado da família quando o cuidador for incorporado ao processo de trabalho das equipes, e quando lhe for mostrado o seu papel, levando em conta as questões ético-legais existentes no cuidado domiciliar, pois muitas atividades exigem conhecimento científico, e a equipe simplesmente ensina e delega à família.
As atividades desenvolvidas pelos cuidadores informais em domicílio, em determinadas situações, são de alta complexidade e realizadas sem a supervisão de um profissional com competência legal, o que acarreta prejuízo no cuidado ao paciente, uma vez que esses cuidadores não sabem prever situações de risco. Para realizar o cuidado em domicílio, é preciso ter conhecimento técnico e científico (e não um conhecimento do senso comum) para atender as necessidades do paciente (LACERDA; PRZENYCZKA, 2008).

Lacerda (2010) destaca que a autonomia para os indivíduos e familiares terem condições de desenvolver o cuidado em domicílio, em uma situação de adoecimento, é a possibilidade de estar capacitado a realizar a ajuda com o apoio do sistema de saúde, com profissionais que ensinem, orientem e acompanhem. A autonomia intersubjetiva é garantida por relações de afeto/amizade, estima social, reconhecimento de direitos e responsabilidades, e é ameaçada pela desvalorização, por desrespeito e violências. Esses fatores contribuem para a compreensão de fenômenos de desrespeito, difamação e traumas, e apoiam a instituição de cuidados com vistas à integridade e à justiça social (SCHUMACHER; PUTTINI; NOJIMOTO, 2013).

Assim, é necessário que as equipes da ESF realizem um acompanhamento sistemático do cuidado ao idoso e à família, com supervisão, pactuação de objetivos, metas e atribuições entre os envolvidos, para que a família retome o seu papel como provedora de cuidados, contando com a ajuda do sistema de saúde.

\section{Conclusão}

É importante destacar que o envelhecimento populacional e os novos arranjos familiares estão proporcionando a criação de um fenômeno social, através da existência de idosos cuidando de idosos, conforme identificado na pesquisa. E que têm como consequência uma relação que acaba gerando sofrimento, 
adoecimento e vulnerabilidade para ambos.

Evidenciou-se, também, no estudo, a fragilidade das ações de apoio/suporte às famílias desenvolvidas pelas equipes da ESF, principalmente as voltadas para os cuidadores, os quais exercem essa atividade sem ter recebido nenhum treinamento, utilizando um conhecimento empírico, de forma solitária e desgastante. Assim, é necessário que os profissionais envolvam os cuidadores nos seus planos de cuidados, tendo este como principal ator para a $\mathrm{AD}$.

Esta pesquisa proporcionou uma caracterização dos cuidadores de idosos para o sistema de saúde de Sobral (CE) e trouxe algumas contribuições para a gestão da ESF, tais como a necessidade de sensibilização dos profissionais sobre a relevância e as especificidades do cuidado domiciliar ao idoso e ao cuidador, exigindo o desenvolvimento de estratégias de apoio e capacitação para cuidadores e família.

Conhecendo as especificidades sociodemográficas e de saúde dos cuidadores de idosos em AD na ESF, e as características desse trabalho, podem-se direcionar políticas públicas de saúde efetivas, que contemplem suas potencialidades e limitações, proporcionando um envelhecimento ativo e uma atenção que potencialize a autonomia dos sujeitos envolvidos entre eles, idosos, cuidadores e profissionais de saúde.

\section{Colaboradores}

MUNIZ, E. A. e FREITAS, C. A. S. L. contribuíram para a concepção do objeto e para a análise e a interpretação dos dados; OLIVEIRA, E. N. e LACERDA, M. R. contribuíram na revisão crítica do conteúdo e na aprovação da versão final do manuscrito.

\section{Referências}

BRASIL. Ministério da Saúde. Portaria GM/MS nº 800, de novembro de 2004. Política Nacional de Saúde do Trabalhador para o SUS. Diário Oficial [da] União. Brasília, DF: Ministério da Saúde, 2004.

Ministério da Saúde. Portaria GM/MS no 2.528, de 19 de outubro de 2006. Política Nacional de Saúde da Pessoa Idosa - PNSPI. Diário Oficial [da] União. Brasília, DF: Ministério da Saúde, 2006a.

Ministério da Saúde. Secretaria de Atenção

à Saúde. Departamento de Atenção Básica. Caderno

de atenção domiciliar, v. 1. Brasília, DF: Ministério da

Saúde, 2012.

\footnotetext{
—. Ministério da Saúde. Secretaria de Atenção

à Saúde. Departamento de Atenção Básica.

Envelhecimento e saúde da pessoa idosa. Brasília, DF:

Ministério da Saúde, 2006b.

DE VALLE-ALONSO, M. J. et al. Sobrecarga y burnout en cuidadores informales del adulto mayor. Enferm.

Univ, México, v. 12, n. 1, p. 19-27, mar. 2015.

DEL DUCA, G. F.; THUME, E.; HALLAL, P. C.

Prevalência e fatores associados ao cuidado domiciliar a idosos. Rev. Saúde Pública, São Paulo, v. 45, n. 1, p.

113-120, fev. 2015.
} 
GRATÃO, A. C. M. et al. Dependência funcional de idosos e a sobrecarga do cuidador. Rev. Esc. Enferm. USP, São Paulo, v. 47, n. 1, p. 137-144, fev. 2013.

\section{GUTIERREZ, D. M. D.; MINAYO, M. C. S. Produção} de conhecimento sobre cuidados da saúde no âmbito da família. Ciênc. Saúde Coletiva, Rio de Janeiro, v. 15, supl. 1, jun. 2010.

\section{HOI, L. V.; THANG, P.; LINDHOLM, L. Elderly} care in daily living in rural Vietnam: need and its socioeconomic determinants. BMC Geriatrics, Londres, v. 11, n. 81, p. 1-10, 2011.

LACERDA, M. R. Cuidado domiciliar: em busca da autonomia do indivíduo e da família - na perspectiva da área pública. Ciênc. Saúde Coletiva, Rio de Janeiro, v. 15, n. 5, p. 2621-2626, ago. 2010.

LACERDA, M. R.; PRZENYCZKA, R. A. Exercício (i) legal da enfermagem: a realidade do cuidador informal. Cogitare Enferm., Curitiba, v. 13, n. 3, p. 543-551, jul./ set. 2008.

MENDOZA-SUAREZ, G. Síndrome de sobrecarga en familiares encargados del cuidado de pacientes con enfermedad neurológica crónica. Rev. Soc. Peru Med. Interna, Lima, v. 27, n. 1, p. 12-18, 2014.

MOTTA, L. B.; AGUIAR, A. C.; CALDAS, C. P. Estratégia Saúde da Família e a atenção ao idoso: experiências em três municípios brasileiros. Cad. Saúde Pública, Rio de Janeiro, v. 27, n. 4, p. 779-786, abr. 2011.

MUNIZ, E. A. Atenção domiciliar na Estratégia Saúde da Família: análise das perspectivas de idosos, cuidadores e profissionais. 2015. 102 f. Dissertação (Mestrado Acadêmico em Saúde da Família) Universidade Federal do Ceará, Sobral, 2015.

PIMENTA, G. M. F. et al. Perfil do familiar cuidador de idoso fragilizado em convívio doméstico da grande Região do Porto, Portugal. Rev. Esc. Enferm. USP, São Paulo, v. 43, n. 3, p. 609-614, set. 2009.

RESENDE, M. C. F.; DIAS, E. C. Cuidadores de idosos: um novo / velho trabalho. Physis, Rio de Janeiro, v. 18, n. 4 , p. 785-800, 2008.

SCHUMACHER, A. A.; PUTTINI, R. F.; NOJIMOTO,

T. Vulnerabilidade, reconhecimento e saúde da pessoa idosa: autonomia intersubjetiva e justiça social. Saúde em Debate, Rio de Janeiro, v. 37, n. 97, p. 281-293, jun. 2013.

SEIMA, M. D.; LENARDT, M. H.; CALDAS, C. P. Relação no cuidado entre o cuidador familiar e o idoso com Alzheimer. Rev. Bras. Enferm., Brasília, DF, v. 67, n. 2, p. 233-240, abr. 2014.

SIMÃO, V. M.; MIOTO, R. C. T. O cuidado paliativo e domiciliar em países da América Latina. Saúde em Debate, Rio de Janeiro, v. 40, n. 108, p. 156-169, mar. 2016.

SOBRAL. Prefeitura Municipal de Sobral. Sobral OnLine, 2012. Disponível em: <http://www.sobral.ce.gov. br>. Acesso em: 1 nov. 2012.

STACKFLETH, R. et al. Sobrecarga de trabalho em cuidadores de idosos fragilizados que vivem no domicílio. Acta Paul Enferm., São Paulo, v. 25, n. 5, p. 768-774, 2012.

VERAS, R. Envelhecimento populacional contemporâneo: demandas, desafios e inovações. Rev Saúde Pública, São Paulo, v. 43, n. 3, p. 548-554, 2009.

VON KANEL, R. et al. Regular physical activity moderates cardiometabolic risk in Alzheimer's caregivers. Med. Sci. Sports Exerc., Bethesda, v. 43, n. 1, p. 181-189, 2011.

WANG, Y. N. et al. Reconciling work and family caregiving among adult-child family caregivers of older people with dementia: effects on role strain and depressive symptoms. J. Adv. Nurs., Malden, v. 67, n. 4, p. 829-840, 2011.

Recebido para publicação em março de 2016

Versão final em maio de 2016

Conflito de interesses: inexistente

Suporte financeiro: não houve 